\title{
Congenital Central Overinflation with Mainstem Bronchial Stenosis: Pre- and Postnatal Imaging and Outcome-Case Report and Brief Review of Literature
}

\author{
Swapnil Sheth ${ }^{1}$ Samarjit S. Ghuman ${ }^{2}$ Tarvinder B.S. Buxi ${ }^{2} \quad$ Seema Sud $^{2}$ Satish Kumar Aggarwal ${ }^{3}$ \\ Anil Sachdev 4
}

${ }^{1}$ Department of Radiology, Sir Gangaram Hospital, New Delhi, India

${ }^{2}$ Department of CT \& MRI, Sir Gangaram Hospital, New Delhi, India

Address for correspondence Swapnil Sheth, DNB, Department of

3 Department of Pediatric Surgery, Sir Gangaram Hospital, New Delhi, Radiology, Sir Gangaram Hospital, Old Rajinder Nagar, New Delhi 110 India

${ }^{4}$ Department of Pediatrics, Sir Gangaram Hospital, New Delhi, India 060, India (e-mail: dr.swapnilsheth4284@gmail.com).

Indian J Radiol Imaging 2021;31:1031-1035.

\begin{abstract}
Congenital overinflation of lung is underdiagnosed prenatally as the imaging features of the same are not well described. We describe a very rare case of central variety of congenital overinflation, secondary to right mainstem bronchial stenosis, not previously described in our knowledge, which presented as an enlarged homogenous

Keywords

- bronchial stenosis

- congenital overinflation

- MRI

- prenatal

- ultrasound echogenic/hyperintense lung with cardio-mediastinal shift and was misdiagnosed as congenital pulmonary airway malformation (CPAM). We reviewed imaging features helpful in prenatal diagnosis of this condition on ultrasound and magnetic resonance imaging and discussed an approach for differential diagnosis. The congenital central overinflation may be considered in prenatal detected echogenic lung lesions with the absence of cysts or systemic arterial supply and presence of hypervascularity or dilated bronchi within the lesion.
\end{abstract}

\section{Introduction}

Prenatal detection of an enlarged echogenic/hyperintense lung on ultrasound or magnetic resonance imaging (MRI) comprises of various differentials like congenital overinflation (CO), bronchial atresia (BA), congenital pulmonary airway disease (CPAM), bronchopulmonary sequestration (BPS), and congenital high airway obstruction syndrome (CHAOS). ${ }^{1,2}$ Imaging features of CPAM and BPS are well documented, however, there is sparse literature about the prenatal imaging findings of $\mathrm{CO}$. $\mathrm{CO}$ is overdistention of alveoli without any parenchymal destruction secondary to bronchial obstruction, from any cause. CO has an incidence rate of 1 in 20,000 to 30,000 live births. ${ }^{1}$

\section{Case Report}

A 30-year-old woman, primigravida with nonconsanguineous marriage and negative family history, came for ultrasound (US) anomaly scan at 19 weeks of gestation with a negative firsttrimester scan. US ( - Fig. 1) revealed a leftward cardiac shift with enlarged echogenic right lung with maintained architecture without focal lung parenchymal lesion or mediastinal mass lesion. The left lung was small in size and appeared less published online

November 30, 2021
DOI https://doi.org/ 10.1055/s-0041-1739380. ISSN 0971-3026.

\footnotetext{
(C) 2021. Indian Radiological Association. All rights reserved. This is an open access article published by Thieme under the terms of the Creative Commons Attribution-NonDerivative-NonCommercial-License, permitting copying and reproduction so long as the original work is given appropriate credit. Contents may not be used for commercial purposes, or adapted, remixed, transformed or built upon. (https://creativecommons.org/ licenses/by-nc-nd/4.0/)

Thieme Medical and Scientific Publishers Pvt. Ltd., A-12, 2nd Floor, Sector 2, Noida-201301 UP, India
} 


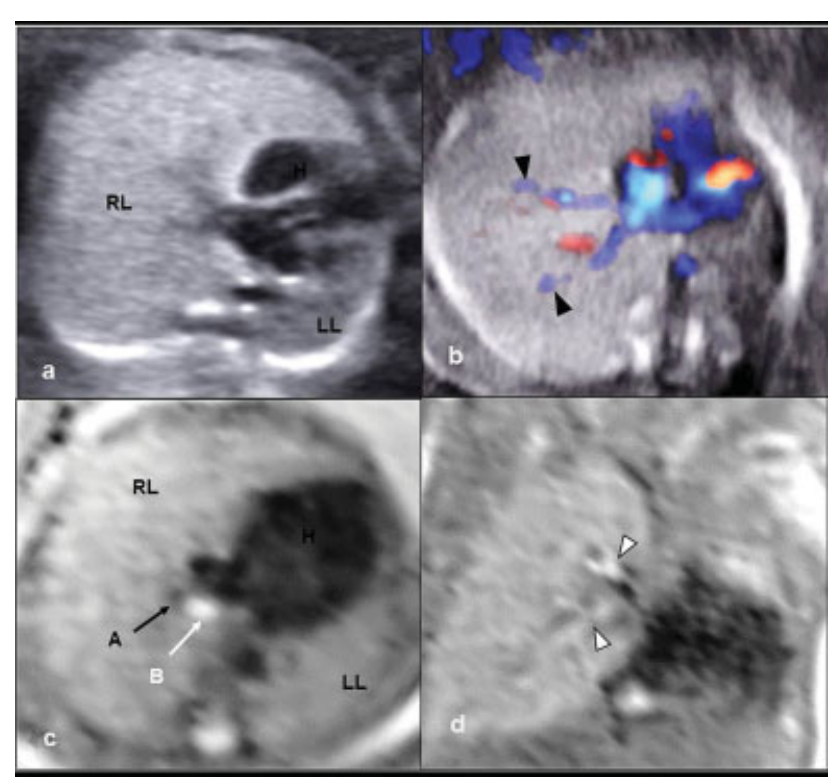

Fig. 119 + weeks: Axial ultrasound of the fetal chest (a) shows enlarged/echogenic right lung (RL), hypoplastic left lung (LL), and leftshifted heart $(\mathrm{H})$. (b) Axial color doppler image shows hypervascularity extending beyond mid of right lung (black arrowhead) on high PRF $(2,000 \mathrm{~Hz})$. (c) Axial HASTE MR image at the right hilum, in addition to ultrasound findings, shows dilated bright oval bronchus (b) at the right hilum (white arrow), larger than arterial (a) flow void (black arrow), and is not seen at the left hilum. (d) HASTE coronal image shows branching tubular dilated bronchi at right hilum (white arrowhead).

echogenic as compared with the right lung. Doppler evaluation ( - Fig. 1) revealed increased vascularity extending just beyond the midportion of the right lung, even on high pulse repetition frequency (PRF) of 2,000 Hz. Possibility of right lung CPAM and hypoplasia/aplasia of the left lung was considered, and fetal MRI was suggested for further evaluation. MR imaging (-Fig. 1) was performed with orthogonal half-Fourier-acquired single-shot turbo spin echo (HASTE) sequences and revealed left cardiac shift; enlarged, morphologically normal, mildly hyperintense leftward herniating right lung, with prominent right lung vascular flow voids, compared with the left lung. The left lung was small/hypoplastic. HASTE hyperintense tubular branching structures, larger than adjacent vascular flow voids were seen at the right hilum extending into the parenchyma, not seen on the contralateral side. Possibility of Type III CPAM or CO right lung and left lung hypoplasia was suggested, and termination of pregnancy was offered but not accepted. The findings were persistent on multiple follow-up US scans (-Fig. 2), with the increased congenital thoracic malformation volume ratio (CVR) from 2.3 at 31 weeks gestation to 6.6 at 36 weeks gestation, with increased mediastinal shift and flattening of the hemidiaphragm, however, without the development of hydrops, which was highly unusual. Fetal MRI at 36 weeks ( - Fig. 2) confirmed the follow-up US findings, and the prominent vascular flow voids of the right lung were now seen more evidently extending up to subpleural parenchyma. Note was made of more hyperintense right middle and lower lobes as compared with the right upper lobe.

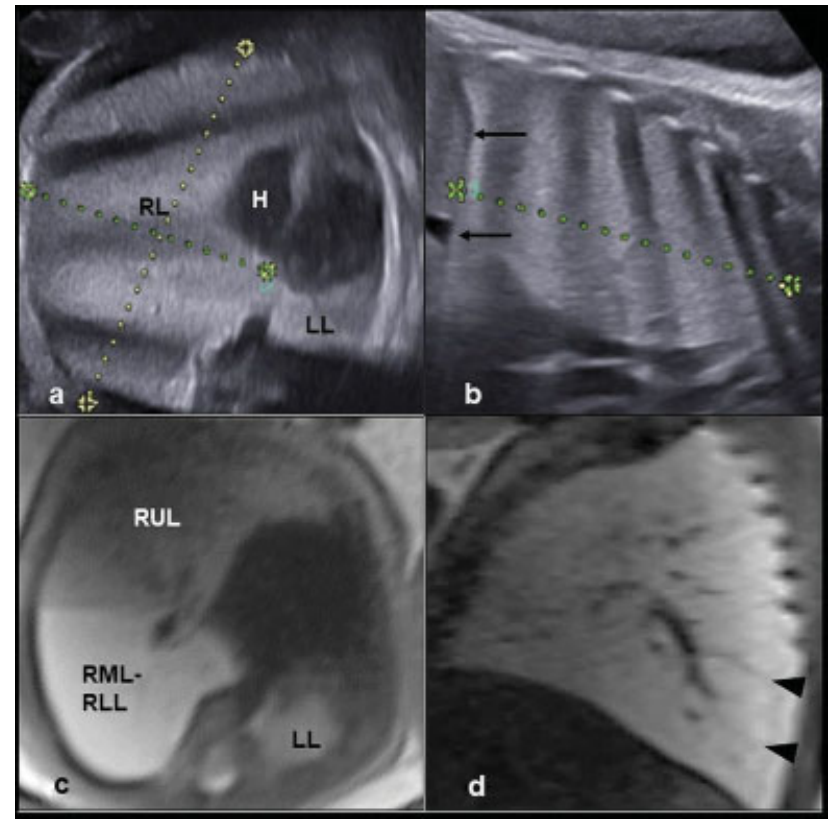

Fig. 2 At 36 + weeks: (a and b) Axial and sagittal ultrasound image respectively reveals an enlarged/echogenic right lung (RL) with leftshifted heart $(\mathrm{H})$, small left lung (LL), flattening of right hemidiaphragm (black arrows). Right lung doppler hypervascularity was seen (not shown). (c) Axial HASTE image reveals more hyperintense middle-lower lobe (RML-RLL), compared with the upper lobe (RUL) of an enlarged right lung. (d) Sagittal HASTE image shows stretched pulmonary vascular flow voids extending up to subpleural lung (black arrowheads).

The male baby was delivered by cesarean section at $38+4$ weeks, cried immediately after birth, with an APGAR score of 6 and 8 at 1 and 5 minutes, respectively, had respiratory distress for which baby required mechanical ventilation. After the abnormal chest radiograph, a contrast chest computed tomography ( - Fig. 3 ) was performed immediately and showed retained fluid within the right middle and lower lobes with intrabronchial fluid and overinflated right upper lobe herniating to the left side. In addition, a tight focal stenosis of proximal right mainstem bronchus without extrinsic compression, with dilated distal bronchi, and normal trachea-carina was seen. The right pulmonary artery and interlobar branches were mildly enlarged with early filling of prominent right pulmonary veins as compared with the left side. The left lung was small/compressed but morphologically unremarkable. The diagnosis of congenital central overinflation (CCO) of the right lung possibly due to right mainstem bronchial stenosis was made. An attempt for the dilatation of the right main bronchus was done with a rigid bronchoscope, however, was not completely successful. On postnatal day 3 , the baby was not maintaining saturation with $100 \%$ of $\mathrm{FiO}_{2}$ on SIMV support, so CT was repeated, which showed complete clearance of retained fluid of right middle and lower lobes, with further overinflation of right lung. In view of the persistent high oxygen requirement and respiratory distress, right pneumonectomy was done on day 3 , but the condition deteriorated with the development of respiratory acidosis, pulmonary arterial hypertension, right ventricular dysfunction, and unfortunately newborn 


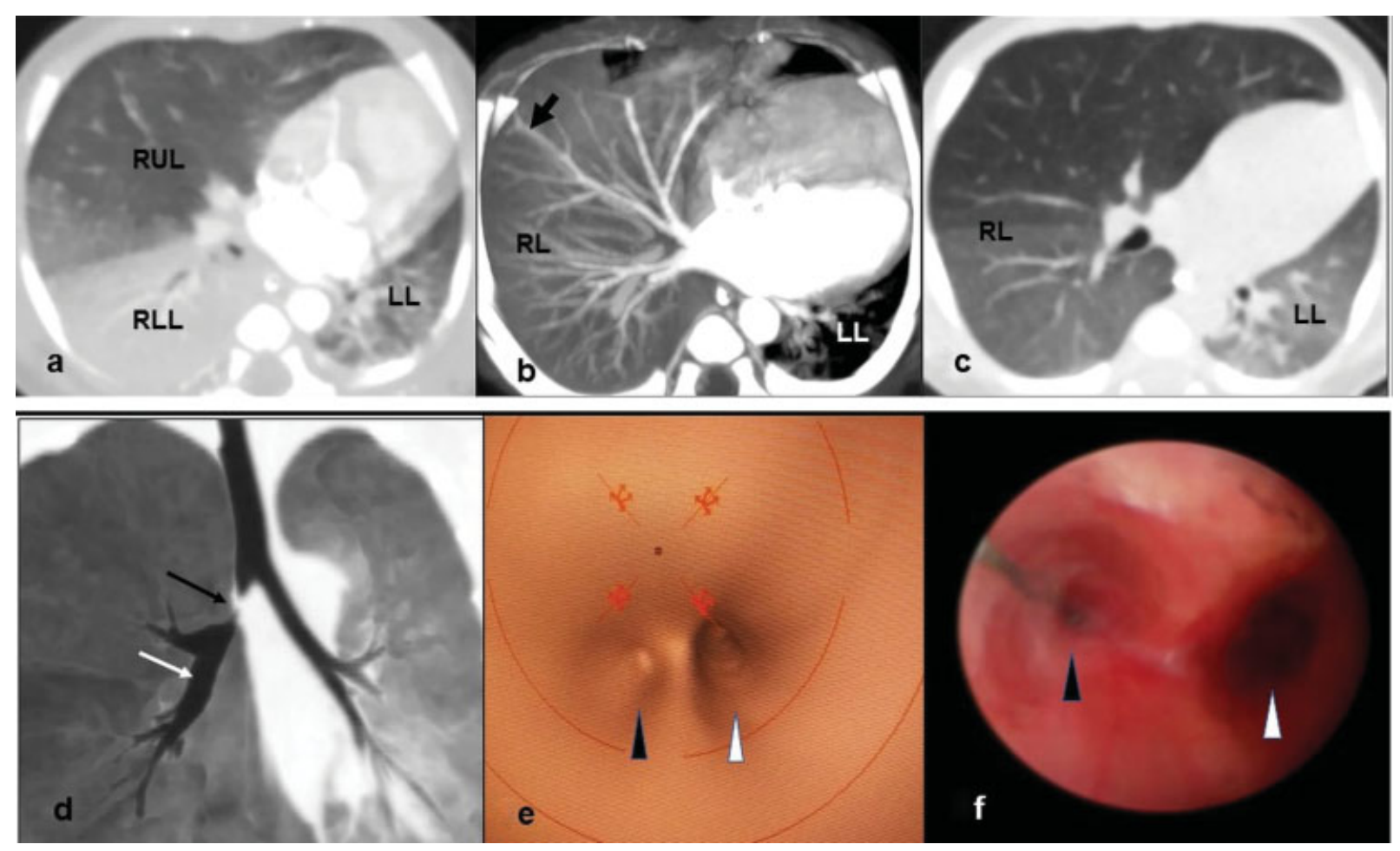

Fig. 3 Postnatal day 1 CECT chest: (a) Axial lung window shows leftward herniating overinflated right upper lobe (RUL), retained fluid in right middle/lower lobes (RML-RLL) and small left lung (LL). (b) Axial maximum intensity projection (MIP) image shows enlarged right pulmonary vessels (thick black arrow). Severe stenosis of proximal right mainstem bronchus was seen (not shown). (c) Day 3 plain CT chest shows almost complete clearance of retained fluid with overinflated entire right lung (RL). (d) Coronal minimum intensity projection (MinIP) image shows proximal right mainstem bronchus stenosis (black arrow) with dilated distal bronchi (white arrow). (e) Virtual and (f) fiberoptic bronchoscopy reveals proximal right mainstem bronchus stenosis (black arrowhead) and normal origin of left mainstem bronchus (white arrowhead).

succumbed on day 5 secondary to hypoxic respiratory failure and shock. The histopathology of the right lung revealed emphysematous changes with normal bronchi and there was no associated polyalveolar lobe.

\section{Discussion}

There are overlapping features and nomenclature of the various thoracic malformations, hence, the common term congenital thoracic malformations (CTM). ${ }^{3}$ The site, time, and severity of airway obstruction play a key role in the formation of a type of CTM. BA in early gestation predisposes the formation of CPAM or bronchogenic cyst, whereas airway obstruction at 16 to 18 gestational weeks may predispose the development of BPS or $\mathrm{CO}^{4}$ The differentials for an enlarged echogenic or hyperintense lung on prenatal US or MRI, respectively include CO, CPAM, BPS, CHAOS, and BA.

\section{Congenital Overinflation}

$\mathrm{CO}$ is also known by congenital alveolar overdistention or congenital large hyperlucent lobe or congenital lobar emphysema, the latter being an inaccurate term, as CO is alveolar overdistention without parenchymal destruction or sometimes polyalveolar lobe..$^{5}$ The cause is thought to be partial or total bronchial obstruction, secondary to either intrinsic causes like maldeveloped cartilage, stenosis/web, mural thickening by cytomegalovirus infection or extrinsic compression by mediastinal mass or enlarged pulmonary artery resulting in a one-way valve mechanism with entrapment of fluid (prenatal) or air (postnatal). ${ }^{5}$ CO usually involves a single lobe or very rarely an entire lung due to mainstem bronchus obstruction, when it is called as CCO.

Imaging findings of $\mathrm{CO}$ : The $\mathrm{CO}$ presents as an enlarged, echogenic/hyperintense lung with maintained morphology and vascular architecture. Dilated fluid-filled/T2 hyperintense tubular structures, representing dilated bronchus proximal to the level of bronchial obstruction is considered a reliable marker of CO. ${ }^{1}$ Pulmonary hypervascularity, defined as significant flow beyond the central half of the involved lung even on high PRF $(>1,500 \mathrm{~Hz})$ on the US, when present strongly favors $\mathrm{CO},{ }^{1,6}$ however, the nonhypervascular $\mathrm{CO}$ also exists. ${ }^{6}$ Lung hypervascularity may be appreciated on MRI as prominent stretched out flow voids extending up to subpleural margins. The two most important imaging markers of $\mathrm{CO}$, i.e., dilated bronchi and hypervascularity were present in our case. This is probably the third published case of central $\mathrm{CO}$, as we could find only two cases of central $\mathrm{CO}$ in the literature, secondary to mainstem intrinsic bronchial obstruction in Johnston et al study, both were right-sided and both had fetal demise at 28 weeks. ${ }^{1}$ Another published case of central CO was due to extrinsic bronchial compression by a lymphatic cyst. ${ }^{7}$ The classical postnatal detected CO most commonly involves the left upper lobe, followed by the right middle lobe, right upper lobe, and both lower lobes in decreasing frequency. However, the two major case series of the prenatal detected $\mathrm{CO}$ by 
Johnston et al and Oliver et al observed lower lobe followed by an upper lobe predominance. ${ }^{1,6}$

The CVR (CTM volume ratio), ratio of lung lesion volume to head circumference on the US are prognosticator for cystic lung lesions, where CVR of $>1.6$ have $80 \%$ chance of developing fetal hydrops. ${ }^{3}$ Despite increased CVR of 2.3 at 19 weeks to 6.6 at 36 weeks, our case did not develop hydrops, the reason is still not understood.

The imaging timing is crucial for diagnosis, as CO like other CTM may regress in size and echogenicity/hyperintensity as the pregnancy progresses. One of the possible mechanisms being temporary bronchial obstruction, is relieved by increasing pressure of fluid accumulation in the affected lung, allowing fluid to extricate. ${ }^{1}$

\section{Other Differentials of Bright Lung}

CPAM is the most common prenatal diagnosed CTM, with five pathological types, and the presence of cysts being diagnostic. However, type III microcystic CPAM presents as solid mass/enlarged bright lung and is a close differential for $\mathrm{CO},{ }^{1}$ but maintained lung morphology, hypervascularity and dilated bronchi may favor $\mathrm{CO}$.

BPS is the second most common prenatal diagnosed CTM, with two types, intralobar and extralobar, both common in lower lobes, ${ }^{5}$ appears as a bright solid mass, and diagnosed reliably by identification of systemic vascular supply from the aorta or its branches, however, failure to identify systemic vascular supply does not exclude BPS. ${ }^{3}$

$\mathrm{BA}$ is a focal bronchial interruption with a radiological presentation like $\mathrm{CO}$ with dilated bronchi proximal to atre- $\mathrm{sia}^{2}$ however, contrary to classical CO it is more common in lower lobes. ${ }^{1}$ Second, the ball valve mechanism does not work in BA as the affected bronchus is occluded instead of narrowed, thus it shows a lesser degree of hyperinflation as compared with $\mathrm{CO} .{ }^{5}$ BA can be proximal (mainstem or lobar bronchi) or peripheral (segmental bronchi).

CHAOS is defined by laryngeal/tracheal obstruction resulting into overdistended and bright lungs bilaterally, flattening or inversion of diaphragm with centrally placed heart and hydrops due to compromised venous return. ${ }^{5}$ Fluid-filled trachea-central bronchi seen on MRI helps to decide the level of obstruction and accordingly surgical planning.

-Table 1 summarizes the findings of various hyperechoic/hyperintense congenital lung malformations and -Fig. 4 shows an approach to these lesions. However, it should be noted that there can be coexistence of multiple lesions in the lung, e.g., CPAM with CO or CPAM with BPS, and these may change the imaging appearances. ${ }^{4}$

Apart from the above discussed congenital lung lesions, the differential for prenatal detected cardio-mediastinal shift also includes lung underdevelopment spectrum, like aplasia, agenesis, or hypoplasia; and doppler may help in diagnosis by demonstrating absent vasculature in lung aplasia and agenesis. Lung hypoplasia is usually secondary to other causes like congenital diaphragmatic hernia (most common), CTM, mediastinal mass, eventration of diaphragm or oligohydramnios, which have their own characteristic imaging appearances. Very rarely, lung hypoplasia can be idiopathic.

Table 1 Differential diagnosis of prenatal detected hyperechoic/hyperintense fetal lung lesion

\begin{tabular}{|c|c|c|c|c|}
\hline & CHAOS & CPAM & BPS & $\mathrm{CO}$ \\
\hline Laterality & Bilateral & $\begin{array}{l}\text { Unilateral } \\
\text { (rarely bilateral) }\end{array}$ & Unilateral & Unilateral (rarely bilateral) \\
\hline Extent of involvement $^{1,5}$ & Both lungs & $\begin{array}{l}\text { Single lobe, rarely } \\
\text { entire lung }\end{array}$ & Single lobe & $\begin{array}{l}\text { Single/bilateral multiple } \\
\text { lobes/rarely entire lung }\end{array}$ \\
\hline Heart position & Central & Eccentric $^{\mathrm{b}}$ & Eccentric $^{b}$ & Eccentric $^{\mathrm{b}}$ \\
\hline Airway ${ }^{1,5}$ & $\begin{array}{l}\text { Dilated fluid-filled } \\
\text { bronchus and } \\
\text { trachea up to level } \\
\text { of obstruction }\end{array}$ & - & - & $\begin{array}{l}\text { Dilated fluid-filled bronchi } \\
\text { within the lesion }\end{array}$ \\
\hline Lung parenchymal cysts ${ }^{5}$ & Absent & Present (except Type 3) & $\begin{array}{l}\text { Absent } \\
\text { (may }+ \text { in } \\
\text { hybrid lesions) }\end{array}$ & Absent \\
\hline Doppler hypervascularity ${ }^{\mathrm{c}, 1,6}$ & Absent & Absent & Absent & Present \\
\hline $\begin{array}{l}\text { Prominent vascular } \\
\text { flow voids on } \mathrm{MRI}^{\mathrm{d}, 6}\end{array}$ & Absent & Absent & Absent & Present \\
\hline Systemic arterial supply ${ }^{5}$ & Absent & $\begin{array}{l}\text { Absent (may }+ \text { in } \\
\text { hybrid lesion) }\end{array}$ & Present & Absent \\
\hline
\end{tabular}

Abbreviations: BPS, bronchopulmonary sequestration; CHAOS, congenital high airway obstruction syndrome; CO, congenital overinflation; MRI, magnetic resonance imaging.

${ }^{a}$ Central overinflation involves an entire lung.

bhen large enough these lesions can cause cardio-mediastinal shift. ${ }^{5}$

'Doppler hypervascularity is described as flow beyond the central part of the affected lung on high pulse repetition frequency $(>1,500 \mathrm{mHz}){ }^{6}$

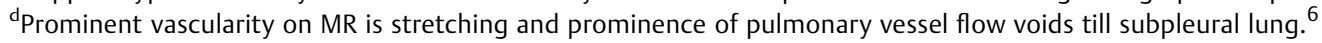




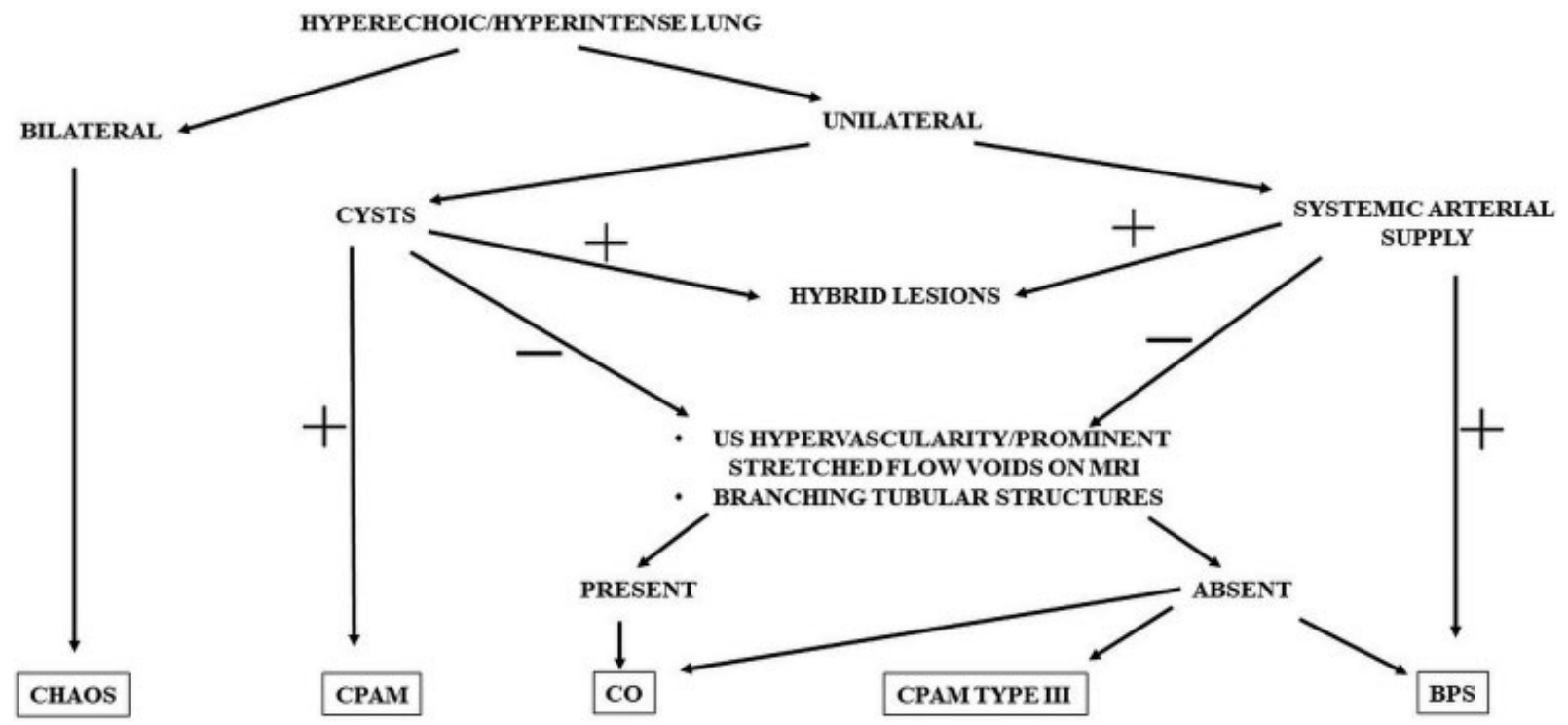

Fig. 4 An approach to a prenatal detected hyperechoic/hyperintense lung (bright lung).

\section{Management and Prognosis}

Symptomatic CO lesions are usually surgically treated, however, a conservative approach may be acquired in asymptomatic or minimally symptomatic patients. ${ }^{1}$ Single lobe or bilateral multiple lobes CO carries a good prognosis, while central CO carries a guarded prognosis. ${ }^{1}$ The prognosis of CHAOS is grave, while BPS and CPAM without hydrops carry a good prognosis.

\section{Conclusion}

In conclusion, differential diagnosis of prenatal detected unilateral enlarged bright lung includes central CO, especially when abnormal lung shows maintained morphology, absent cysts or systemic vascular supply, dilated fluid-filled central bronchi, doppler hypervascularity on high PRF $(>1,500 \mathrm{~Hz})$, diagnosis of $\mathrm{CO}$ can be made reliably. The central $\mathrm{CO}$ carries a guarded prognosis and may be considered in cases having high CVR without hydrops, as occurred in our case.

Financial Support and Sponsorship

None.
Conflict of Interest

None declared.

\section{References}

1 Johnston JH, Kline-Fath BM, Bitters C, Calvo-Garcia MA, Lim FYY. Congenital overinflation: prenatal MRI and US findings and outcomes. Prenat Diagn 2016;36(06):568-575

2 Kozaki M, Iraha Y, Masamoto H, et al. Fetal congenital peripheral bronchial atresia diagnosed by magnetic resonance imaging: two case reports. AJP Rep 2018;8(04):e201-e205

3 Annunziata F, Bush A, Borgia F, et al. Congenital lung malformations: unresolved issues and unanswered questions. Front Pediatr 2019;7:239

4 Kunisaki SM, Fauza DO, Nemes LP, et al. Bronchial atresia: the hidden pathology within a spectrum of prenatally diagnosed lung masses. J Pediatr Surg 2006;41(01):61-65, discussion 61-65

5 Biyyam DR, Chapman T, Ferguson MR, Deutsch G, Dighe MK. Congenital lung abnormalities: embryologic features, prenatal diagnosis, and postnatal radiologic-pathologic correlation. Radiographics 2010;30(06):1721-1738

6 Oliver ER, DeBari SE, Horii SC, et al. Congenital lobar overinflation: a rare enigmatic lung lesion on prenatal ultrasound and magnetic resonance imaging. J Ultrasound Med 2019;38(05): 1229-1239

7 Man DW, Donell ST, Raafat F, Eckstein HB. Congenital obstructive emphysema of the left lung associated with a mediastinal lymphatic cyst. Thorax 1984;39(03):211-212 